\title{
Transcriptional Regulation of the BCL-6 Gene: Mechanistic Dissection Using Mutant Cell Lines
}

\author{
Gang Zhou and Santa Jeremy Ono \\ Department of Immunology, University College London, University of London, Institutes of Ophthalmology \\ and Child Health,
}

Accepted August 27, 2002

\begin{abstract}
Background: Mice lacking the BCL-6 gene product exhibit overexpression of $\mathrm{T}$ helper cell type 2 cytokines, and multi-organ inflammatory responses characterized by eosinophilia and infiltration by IgE + B lymphocytes. Elevated IgE levels appear to result from loss of BCL-6's role in repression of the Stat6-dependent processes of I- epsilon transcription and IgE class-switching. Understanding the regulation of the BCL- 6 gene expression is therefore relevant to the allergic response, due to the IgEdependent activation of mast cells and basophils.

Materials and Methods: We analyzed a pair of isogenic cell lines that exhibit differential expression of the BCL-6 gene and mapped the control point to transcription using nuclear run-on assays. Direct analyses of the BCL- 6 gene and transfection experiments were carried out to map the molecular basis for the differential expression.

Results: In this study, we report that pair of isogenic cell lines exhibits differential expression of BCL-6. BCL-6 mRNA is barely detectable in Jijoye, a Burkitt lymphoma $B$ cell line, but is abundant in Clone-13, a mutant cell line
\end{abstract}

derived from Jijoye. Corresponding to the mRNA level, BCL-6 protein is detected only in Clone-13, but not in Jijoye. Nuclear run-on assays indicate that BCL-6 expression is regulated mainly at the transcriptional level in these two cell lines. Gene structural alterations are not detected in a region including a $3-\mathrm{kb}$ promoter and the first intron, where most of the chromosomal translocations are observed in diffuse large cell lymphoma (DLCL). Interestingly, multiple, heterozygous point mutations are identified in the first intron, the hypermutation region of BCL-6, in both of the two cell lines. However, in transient transfection experiments these mutations have no effects on gene expression.

Conclusions: Our data suggest that the distinct profile of BCL-6 expression in Jijoye/Clone-13 is due to either a missing negative element in a different portion of the gene or that there is an issue of chromatin accessibility operating in BCL-6 gene regulation. This pair of isogenic cell lines (Jijoye/Clone-13) thus provides a new system to dissect the regulation of BCL-6 gene expression.

\section{Introduction}

The BCL-6 gene encodes for a transcriptional repressor that contains six Kruppel-like zinc fingers at its carboxyl-terminus (1-3), and an N-terminal POZ domain homologous to members of zinc finger transcription factors (4-8). Previous studies have shown that BCL-6 mRNA is readily detectable in most mature B-cell lines and many tissues, and at low levels in $\mathrm{T}-$, myeloid, and erythroid cells $(9,10)$. Immunohistologic experiments demonstrate that there is strong nuclear expression of BCL-6 protein in follicular lymphomas, diffuse large B-cell lymphomas, Burkitt's lymphomas, and nodular, lymphocyte-predominant Hodgkin's disease, with the highest levels in germinal center B lymphocytes $(11,12)$. Transient transfection assays indicate that BCL-6 can function as a potent sequence-specific transcriptional repressor through its consensus DNA binding sequence $(13,14)$,

Address correspondence and reprint requests to: Santa Jeremy Ono, GlaxoSmithKline Professor of the Biomedical Sciences, University College London, University of London, Institutes of Ophthalmology and Child Health, 11-43 Bath Street, London, ECIV 9EL, United Kingdom. Phone: 01 1-44-207-608-4069; fax: 011-44-207-608-4044; e-mail: santa.ono@ucl.ac.uk. which resembles the binding sites for several STAT factors (15). Chromosomal translocations resulting in promoter substitutions of the BCL- 6 gene recurrently occur in the diffuse large cell lymphoma (DLCL), a subtype of non-Hodgkin's lymphomas $(16,17)$. These rearrangements include replacements of the $5^{\prime}$ regulatory region of the BCL- 6 gene with regions of the immunoglobulin gene locus or other translocation partners, while invariably leaving the BCL-6 coding region intact (16-20). Besides frequent chromosomal translocations, somatic hypermutation similar to Ig gene is observed in the first intron of the BCL-6 gene $(21,22)$. Mice with a disrupted BCL- 6 gene do not develop germinal centers and have increased expression of Th2 cytokines $(15,23)$. These findings strongly suggest that BCL-6 play critical roles in regulating B- and T-cell responses, and that its deregulated expression may contribute to lymphomagenesis.

Despite a great deal of work focused on this gene since 1993, its precise biological function and transcriptional regulation are poorly understood. In this study, we report the differential expression of BCL-6 in a pair of isogenic human B lymphoblastoid cells, Jijoye and Clone-13, as determined first by mRNA 
differential display. RT-PCR and Northern blot analysis further verify that BCL-6 mRNA is readily detectable in Clone-13, the mutant cell line, but only barely detected in its parental cell line, Jijoye. Corresponding to the mRNA level, BCL-6 protein is only detected in Clone-13, but not in Jijoye. Nuclear runon assays indicate that BCL-6 gene expression is mainly regulated at the transcriptional level in the two cell lines. Whereas multiple sequence differences are identified in the first introns of the BCL- 6 genes within the cell lines, these mutations do not have significant effects on the expression of the downstream reporter gene. Our results indicate that the distinct expression of the BCL-6 gene in Jijoye/Clone-13 is not due to mutations in the first intron or promoter substitutions caused by chromosomal translocations. It is more likely that the differences are due to defects in trans-acting factor(s). This pair of isogenic B-cell lines therefore provides an important system for understanding the regulation of BCL-6 gene expression.

\section{Materials and Methods}

\section{Construction of Plasmids}

The pGL2-Control Vector (Promega, Madison, WI, USA) with an SV40 promoter and enhancer was used in transfection experiments. The promoterless plasmid pGL2-Basic (Promega) was included as background. A 4.7-kb Kpn I-Bgl II fragment from pLA/B14.5 was ligated into pGL2 Basic to generate plasmid BlucKp/Bg. Plasmids J4, J6, C1, and C2 were generated by PCR. They have the same promoter region as BlucKp/Bg; the first exon and part of the first intron were amplified from different alleles of Jijoye and Clone-13. J4 and J6 contain fragment derived from the two alleles of Jijoye, respectively. C1 and C2 were derived from Clone-13. The sequences of the oligos used in PCR are as follows (position +1 refers to the first nucleotide of the BCL-6 CDNA):

\section{B6Sl (+297bp-+318bp), 5'-AACTCGAG ACGCTCTGCTTATG-3'; Xho I \\ B6AS 1 (+1419bp-+1441bp), 5'-AGGA AGATCTCGGCTCTGAAAGG-3'; Bgl II}

One or two bases in each oligonucleotide were changed to create a restriction site for cloning. PCR products were purified with Qiaquick Gel Extraction Kit (Qiagen, Crawley, West Sussex, U.K.), digested with Xho I and Bgl II, and then subcloned into the Xho I-Bgl II site of plasmid BlucKp/Bg. The sequences of the plasmids were confirmed by dideoxy sequencing.

\section{Cell Culture and Transient Transfection}

Raji, Jijoye, and Clone-13 cells were maintained in RPMI 1640 supplemented with $10 \%$ FBS, 2 mM glutamine, and $1 \mu \mathrm{g} / \mathrm{ml}$ each of penicillin and streptomycin. Raji is a Epstein-Barr virus-transformed human B lymphoblastoid cell line. Jijoye cells (originally from a Burkitt's lymphoma patient) were originally isolated by Pulvertaft and Pulvertaft (24) and designated as P3. Hinuma et al. (25) then isolated subclones of the line in semisolid media and generated a line designated p3J-HR-1, which is a high producer of viral capsid antigen. Clone- 13 cells were derived by further subcloning of the p3J-HR-1 cell line with the aim of producing isogenic cell lines with varying efficiencies of conversion, from the latent to lytic cycles of the virus (26).

Ten-microgram reporter DNA and 5- $\mu \mathrm{g}$ pCH110 (Pharmacia, Peapack, NJ, USA) were added to a $0.4-\mathrm{cm}$ electroporation cuvette. Cells $\left(3 \times 10^{6}\right)$ suspended in $300 \mu \mathrm{l}$ complete media were transferred to the cuvette and mixed well with DNA before pulsed at $310 \mathrm{~V}, 1000 \mathrm{uF}$ using an Invitrogen Electroporator II (Invitrogen, Carlsbad, CA, USA). Then cells were incubated at $37{ }^{\circ} \mathrm{C}, 5 \% \mathrm{CO}_{2}$ for $40 \mathrm{hr}$ before harvest for luciferase assay. Luciferase activity was determined using a luminometer (Monolight 2010, Analytical Luminescence Laboratory, Mississauga, Ontario, Canada) following the protocol of Luciferase Assay System (Promega). Relative luciferase values were normalized to $\beta$-gal expression and shown as percentages of the value of pGL2-Control Vector.

\section{mRNA Differential Display Analysis}

Differential display experiments were carried out in a GeneAmp PCR System 2400 (Perkin-Elmer, Norwalk, CT, USA), using a RNAimage Kit (GenHunter, Nashville, TN, USA) according to the manufacturer's instruction. Total RNA was extracted from each cell line using RNA STAT-60 Reagent (TEL-TEST, Friendswood, TX, USA) and then treated with RQ1 RNase-Free DNase I (Promega) to eliminate genomic DNA contamination. The first strand cDNA was synthesized by reverse transcription with H-TIIG anchored primer in the presence of MMLV reverse transcriptase, then amplified with a $5^{\prime}$ arbitrary primer H-AP6 (5'AAGCTTGCACCAT- $3^{\prime}$ ) in the presence of ${ }^{32} \mathrm{P}-\mathrm{dCTP}$ (3000 Ci/mmol; ICN, Costa Mesa, CA, USA) and Taq DNA Polymerase (Promega). The amplified products were resolved on a $6 \%$ polyacrylamide denaturing sequencing gel. After autoradiography, the bands of interest were recovered and reamplified by PCR.

\section{Cloning and Sequence Analysis}

The products of reamplification from differential display were cloned into the vector pUC57/T using the T-cloning Kit (MBI, Vilnius, Lithuania). Four to six independent bacterial colonies were randomly picked up and lysed with the method described by Zhang et al. (27). The lysates were used as templates for PCR amplification in which $\mathrm{Ml3}$ forward and reverse primers flanking the cloning site of the vector were used to amplify the fragments containing the inserts. The PCR fragments were sequenced using Delta Taq Cycle Sequencing Kit (Amersham, Cleveland, $\mathrm{OH}$, USA) according to the manufacturer's specification. 


\section{RT-PCR Assay}

A pair of primers specific to the human BCL-6 CDNA (spanning base pairs +427 to +645 , covering part of exon 3 and exon 4) was synthesized and used in PCR reactions. Total RNAs treated with RQl were reverse transcribed with random primers and $M$ MLV transcriptase (Gibco-BRL, Gaithersburg, MD, USA). Then $1 / 10$ volume of cDNA was used as template for the following PCR amplification. Thirty cycles were run at $94{ }^{\circ} \mathrm{C}$ for $1 \mathrm{~min}, 60^{\circ} \mathrm{C}$ for $2 \mathrm{~min}$, and $72{ }^{\circ} \mathrm{C}$ for $3 \mathrm{~min}$. Twenty microliters of each PCR product was analyzed on $1.5 \%$ agarose gels stained with ethidium bromide. The same cDNAs were used for amplification of GAPDH and HLA-DR $\alpha$ to evaluate the integrity and amount of the RNA samples used in RT-PCR. The sequences of oligonucleotide primers for PCR amplification are as follows:

BCL-6 sense: 5' -GTTGTCATTGTTGTGAGCCG-3'

BCL-6 antisense: 5'-CATCACAGCCATGATGTTGC-3' (219 bp)

DR $\alpha$ sense: 5'-GAGTT CTATCTGAATCCTG-3'

Dr $\alpha$ antisense: 5'-GTTCTGCTGCATTGCTTTTGC-3' (643 bp)

GAPDH sense: 5' -TGATGACATCAAGAAGGTGGT-3'

GAPDH antisense: 5'-CAGTGAGGGTCTCTCTCTTCC-3' (301 bp)

\section{DNA and RNA Analysis}

Northern Blot Analysis Total RNA samples $(20 \mu \mathrm{g}$ per lane) were size fractionated on a $1 \%$ formaldehyde agarose gel and transferred to a Hybond-N+ membrane (Amersham). A 2.0-kb EcoR I fragment derived from the eukaryotic expression vector pMT2T-BCL-6 (10) was used as probe, ${ }^{32} \mathrm{P}$-labeled with MegaPrime DNA Labeling Kit (Amersham) followed by purification with NucTrap Push Columns (Stratagene, La Jolla, CA, USA). Northern blot analysis was performed at $68^{\circ} \mathrm{C}$ using QuikHyb Hybridization Solution (Stratagene) following the manufacturer's protocol.

Southern Blot Analysis Preparation of high-molecular-weight DNA, restriction enzyme digestion and electrophoresis were performed according to standard procedures. Fifteen micrograms of genomic DNA from Jijoye and Clone-13 were digested by Hind III (New England BioLabs, Beverly, MA, USA), fractionated by $0.9 \%$ agarose gel electrophoresis, denatured, and transferred to a Hybond-N+ membrane (Amersham). Probe 1, a 2.9-kb Avr II-Xho I fragment derived from plasmid pLA/B14.5, covers the $\sim 3$-kb 5 '-flanking region. Probe 2, a 4.2-kb fragment digested from pLA/B14.5 by Bgl II, spans through the hot-spot region for chromosomal translocations of the BCL-6 gene. Hybridization was performed under the same condition as Northern blot, except that the temperature was reduced to $65^{\circ} \mathrm{C}$ for hybridization and washing.
Western Blot Analysis Cells $\left(3 \times 10^{6}\right)$ were resuspended in PBS, lysed with $2 \times$ SDS-PAGE sample buffer containing 2-mercaptoethanol, boiled for $5 \mathrm{~min}$, and electrophoresed through a $8 \%$ SDS-PAGE gel. The proteins were then electrotransferred to PVDF membranes (Bio-Rad, Hercules, CA, USA). The proteins were immunostained with antibody Bcl-6 (N-3) (Santa Cruz Biotechnology, Santa Cruz, CA, USA) followed by alkaline phosphatase conjugated anti-Rabbit IgG antibodies. Western blot analysis was carried out using a Western-Light Chemiluminescent Detection System (Tropix, Bedford, MA, USA), following the manufacturer's specifications. After Western blot analysis the membrane was stained with amido black to evaluate the equivalency of protein loading.

\section{Nuclear Run-on Transcription Assay}

Nuclear run-on assays were performed according to the methods described previously with minor modification (28). Briefly, approximately $1 \times 10^{7}$ cells were collected and washed twice with PBS, and incubated with $2 \mathrm{ml}$ lysis buffer (10 mM Tris-Cl, pH7.4, $10 \mathrm{mM}$ $\mathrm{NaCl}, 3 \mathrm{mM} \mathrm{MgCl} 2,0.5 \% \mathrm{NP}-40)$ on ice for $5 \mathrm{~min}$. Nuclei were pelleted for $5 \mathrm{~min}$ at $500 \times \mathrm{g}$. After washing once with the lysis buffer without NP-40, the pellets were resuspended in $100 \mu \mathrm{l}$ of storage buffer (50 mM Tris-Cl, pH8.3, $40 \%$ glycerol, $5 \mathrm{mM} \mathrm{MgCl}_{2}$, $0.1 \mathrm{mM}$ EDTA). One hundred microliters of $2 \times$ reaction buffer ( $10 \mathrm{mM}$ Tris-Cl, pH8.0, $5 \mathrm{mM} \mathrm{MgCl}_{2}, 300$ $\mathrm{mM} \mathrm{KCl}, 5 \mathrm{mM}$ DTT, $1 \mathrm{mM}$ each of ATP, GTP, CTP) and $200 \mu \mathrm{Ci} \alpha-{ }^{32}$ P-UTP $(>800 \mathrm{Ci} / \mathrm{mmol}$; ICN) were added to the nuclei suspension along with $5 \mu \mathrm{l}$ RNasin (Promega). The mixture was incubated at $30{ }^{\circ} \mathrm{C}$ for $30 \mathrm{~min}$. The reaction was terminated by addition of 40 U RQ1 RNase-Free DNAse I (Promega) and further incubated for $10 \mathrm{~min}$. Then Nuclear RNA was isolated with RNA STAT-60 Reagent as described.

Plasmid pHeboMTBCL6 (10) containing the fulllength BCL-6 CDNA, cloning vector pMT2T, and plasmid pSP64- $\gamma$-actin (gift from Dr Tom Maniatis, Harvard University) containing the $\gamma$-actin cDNA were linearized with Kpn I, EcoR I, and EcoR I, respectively. After gel purification, $5 \mu \mathrm{g}$ DNA of each sample was denatured in $0.2 \mathrm{~N} \mathrm{NaOH}$ at $65^{\circ} \mathrm{C}$ for $1 \mathrm{hr}$ and then neutralized with 10 volumes of $6 \times$ SSC. The DNA was spotted onto a Hybond-N+ membrane (Amersham) using a slot blot manifold (Bio-Rad) and then UVcrosslinked. The filters were prehybridized for $16 \mathrm{hr}$ at $65{ }^{\circ} \mathrm{C}$ in prehybridization buffer $(10 \mathrm{mM}$ Tris- $\mathrm{Cl}$, $\mathrm{pH}$ 7.5, $300 \mathrm{mM} \mathrm{NaCl}, 10 \mathrm{mM}$ EDTA, $0.5 \mu \mathrm{g} / \mathrm{ml}$ salmon sperm DNA, $0.05 \%$ sodium pyrophosphate, $2 \times$ Denhardt). Hybridizations were performed with equal amounts of labeled nuclear RNA in hybridization buffer (10 mM Tris-Cl, pH 7.5, $300 \mathrm{mM} \mathrm{NaCl}, 10 \mathrm{mM}$ EDTA, $0.2 \%$ SDS) for $44 \mathrm{hr}$ at $65^{\circ} \mathrm{C}$. The filters were washed twice with $2 \times$ SSC, $0.1 \%$ SDS at room temperature for $10 \mathrm{~min}$, then $0.2 \times$ SSC, $0.1 \%$ SDS for $30 \mathrm{~min}$ at $60{ }^{\circ} \mathrm{C}$. After incubation with $2 \times$ SSC, $10 \mu \mathrm{g} / \mathrm{ml}$ RNase A at $37^{\circ} \mathrm{C}$ for $30 \mathrm{~min}$, the filters were rinsed twice in $2 \times$ SSC before autoradiography. 


\section{Results}

The BCL-6 Gene is Differentially Expressed in Jijoye and Clone-13

We previously described a mutant Burkitt's cell line, Clone-13 (29), where HLA-DP and -DR are not expressed due to mutations in both alleles of the transcription factor CIITA (30), and HLA-DQ is still expressed at a high level. In its parental cell line, Jijoye, with the presence of CIITA, all HLA molecules are expressed. It is now known that CIITA is a critical player in regulating multiple genes involved in antigen presentation (31-33). However, evidence showed that other factors might exist that can also mediate antigen presentation $(30,34)$. To identify other gene products germane to the immune response, we looked for additional genes that were differentially expressed between the Jijoye and Clone-13 cell lines using the technique of mRNA differential display (35).

Sequence analysis of one of the differentially expressed species detected only in Clone-13 (Fig. 1) turned out to completely match the BCL-6 cDNA sequence (from $+3344 \mathrm{bp}$ to the polyA end). The BCL-6 gene, which was cloned from chromosomal translocations affecting band 3q27 (1), encodes for a 95-kD nuclear protein containing six C-terminal Kruppel-like zinc finger motifs (1-3), and an $\mathrm{N}$-terminal POZ domain homologous to members of zinc finger transcriptional factors (4-8).

To verify the results of differential display (which was itself repeated three times with two independent RNA sample preparations), two primers specific for

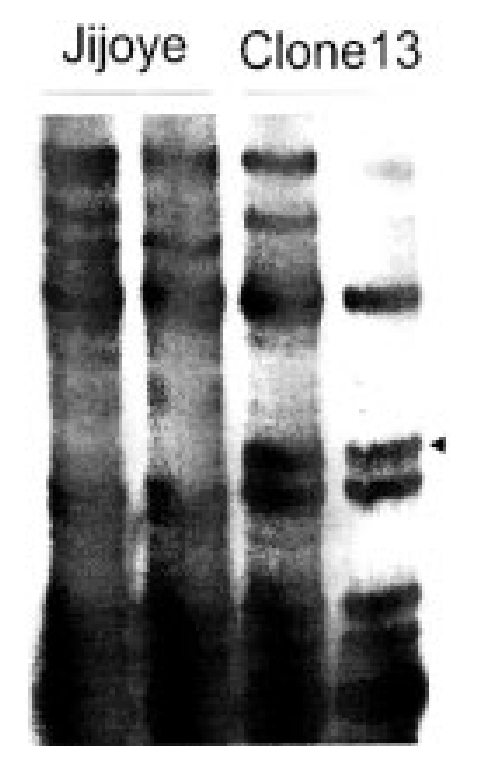

Fig. 1. Differential display of messenger RNA from Jijoye and Clone-13. Total RNA from each cell line was reverse transcribed and amplified by PCR in duplicate, using H-T11G as the anchor primer and H-AP6 (5'-AAGCTTGCACCAT-3') as the arbitrary primer. The radiolabeled PCR products were resolved on a $6 \%$ sequencing gel. The band indicated by the arrowhead was recovered from the gel. Upon sequence analysis, this particular mRNA was determined to be the BCL- 6 mRNA. the human BCL-6 cDNA sequence (spanning base pairs +427 to +645 , covering part of exon 3 and exon 4) were used in RT-PCR to detect BCL-6 mRNA in Jijoye and Clone-13. As shown in Figure 2A, BCL-6 mRNA was easily detected in the mutant cell line, Clone-13, whereas the BCL-6 PCR product in Jijoye was barely detectable. The same results were obtained with different RNA preparations and with another pair of PCR primers amplifying portion of the 3'-UTR (spanning base pairs +1678 to +1894 ; data not shown). Because the chromosomal breakpoints of the BCL- 6 gene rearrangements cluster within a $4-\mathrm{kb}$ region spanning the 5 ' flanking region, the first exon, and the first intron $(1,17)$, leaving the coding domain intact, PCR products corresponding the coding region and 3'-UTR may better ascertain the presence of the BCL- 6 transcripts. As controls, amplification products of HLA-DR $\alpha$ were only detected in Jijoye; products of GAPDH were nearly equivalent in the two cell lines, verifying the phenotype of the cells and controlling for RNA quality and PCR reactions.

Northern blot analysis was carried out using a 2.0-kb EcoR I fragment derived from the BCL-6 cDNA plasmid pMT2T-BCL-6 (10). A band with the expected size $(3.8 \mathrm{~kb})$ was readily detectable in

A.

B.
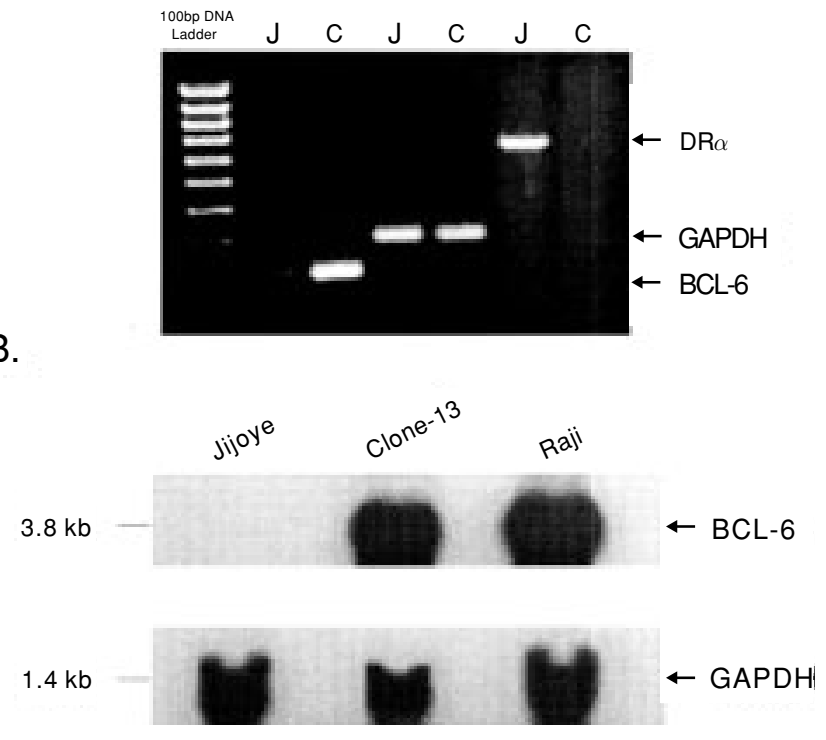

Fig. 2. Comparison of BCL-6 mRNA levels in Jijoye and Clone-13. (A) RT-PCR analysis of BCL-6 mRNA expression in Jijoye and Clone-13. Total RNAs were reverse transcribed with MMLV and random primers; a pair of primers specific for human BCL-6 CDNA was used in the following PCR reactions as described in Material and Methods. GAPDH and DR $\alpha$ were used as controls to evaluate the quality and amount of RNA samples used in RT-PCR. The data are representative of three independent analyses. (B) Northern blot analysis of BCL-6 mRNA in Jijoye, Clone-13 and Raji. Twenty micrograms of total RNA from each cell line was hybridized with a 2.0-kb EcoR I fragment derived from PMT2T-BCL6. The same blots were stripped and rehybridized with the GAPDH probe to evaluate the equivalency of RNA loading in each lane. 
A.

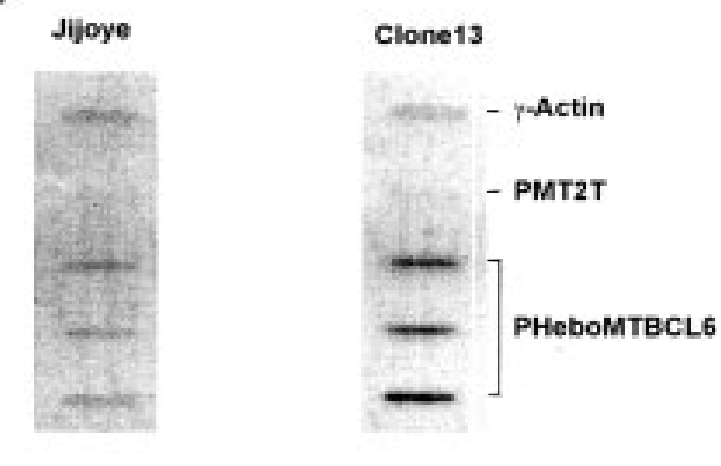

B.

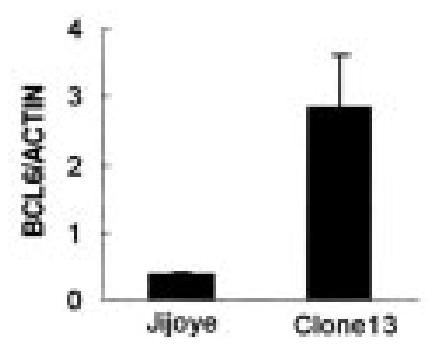

Fig. 3. Western blot analysis of BCL-6 protein expression in Raji, Jijoye, Clone-13 and Jurkat. Cells $\left(3 \times 10^{6}\right)$ were harvested and washed with PBS. Cells were lysed with SDSPAGE sample buffer. Cell lysates from each cell line were analyzed using the anti-Bcl-6 $(\mathrm{N}-3)$ and developed using an ECL kit. A $95-\mathrm{kb}$ band corresponding to the BCL-6 protein was detected only in Raji and Clone-13 cell lines.

Clone-13 (Fig. 2B, lane 2), and the intensity of the band is comparable to that in the BCL-6-positive cell line, Raji (Fig. 2B, lane 3). However, only a trace amount of BCL-6 mRNA was detected in Jijoye (Fig. $2 \mathrm{~B}$, lane 1$)$. We next analyzed the BCL-6 protein levels in the two cell lines by Western blot analysis. BCL-6 proteins were detected in Raji and Clone-13 cells (Fig. 3, lanes 1 and 3, respectively), but not in Jijoye and Jurkat (Fig. 3, lanes 2 and 4, respectively). These results clearly demonstrated that BCL-6 is differentially expressed in the two cell lines.

\section{The BCL-6 Transcription Rate is Higher in Clone-13 than in Jijoye}

The transcription rates of BCL- 6 in these two cell lines were compared by nuclear run-on assay (Fig. $4 \mathrm{~A})$. The intensity of the signals is quantified and shown as relative intensity (Fig. 4B) by subtracting the signal of empty vector pMT2T from plasmid pHeboMTBCL6 containing the full-length BCL-6 cDNA, and then compared to plasmid pSP64- $\gamma$-actin containing the $\gamma$-actin cDNA. The results showed that the transcription rate in Clone-13 is approximately 5 -fold of that in Jijoye (Fig. 4B).

The BCL-6 Gene has no Gross Deletions in the Promoter $(\sim 3 \mathrm{~kb})$ and the First Intron in Jijoye/Clone-13

To determine whether structural alterations may have occurred in the BCL- 6 gene that might explain the distinct BCL-6 expression phenotype of these cells, Southern blot analyses were performed using probes spanning the promoter, first exon, and first intron of the BCL-6 gene (Fig. 5A). This is important in view of the propensity of the BCL- 6 gene to undergo chromosomal translocations. Three bands with the expected size ( $3.7 \mathrm{~kb}, 1.3 \mathrm{~kb}$, and $914 \mathrm{bp})$ were detected by probe 1 (Fig. 5B), four bands $(3.7 \mathrm{~kb}, 1.3 \mathrm{~kb}$, $1.0 \mathrm{~kb}$, and $676 \mathrm{bp}$ ) were detected by probe 2 (Fig. 5C). These data suggest that there are no gross changes in the BCL-6 gene in these cell lines.

\section{Multiple Mutations Cluster in the First Intron of the BCL-6} Gene in both Jijoye and Clone-13

To investigate whether point mutations in the BCL6 gene may explain the higher transcription rate in Clone-13 than Jijoye, we amplified and cloned the hypermutable region of BCL-6 (Fig. 6A) from

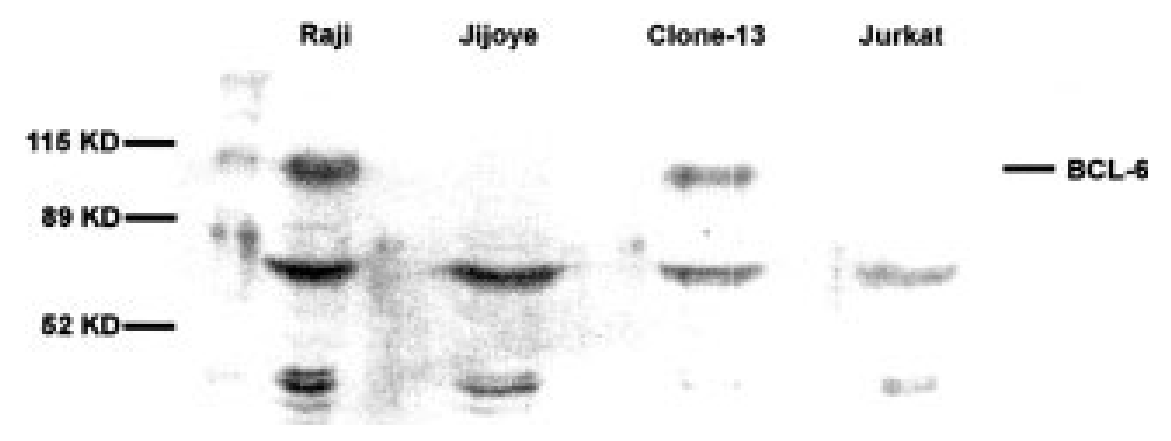

Fig. 4. Comparison of transcriptional rates of BCL-6 in Jijoye and Clone-13 by nuclear run-on assay. (A) Linearized denatured plasmid DNA was fixed on duplicate filters. $\gamma$-Actin cDNA and pMT2T vectors were used as positive and negative controls, respectively. PHeboMTBCL6 was dotted in triplicate on each filter. Nuclei were isolated from Jijoye and Clone-13, and ${ }^{32}$ P-UTP labeled nascent RNAs used in hybridization with the filters. (B) The intensities of signals in A were analyzed with the BDS-Image Analysis System (version 1.3; Biological Detection Systems, Pittsburgh, PA, USA) and quantitated using the NIH Image program (version 1.61). Relative signal density was represented as ratio of BCL-6/ $\gamma$-actin after deduction of the background signal (pMT2T). 
A.

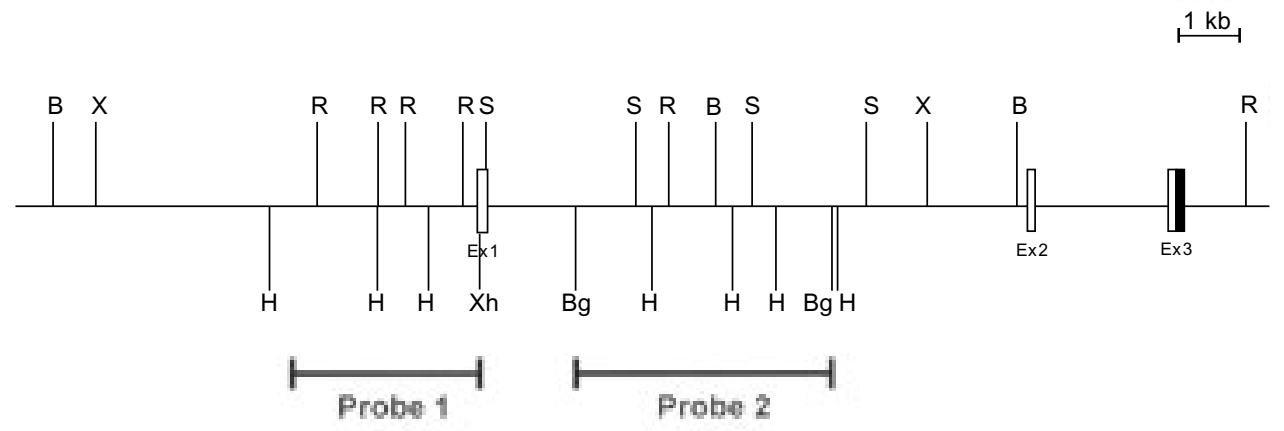

B.

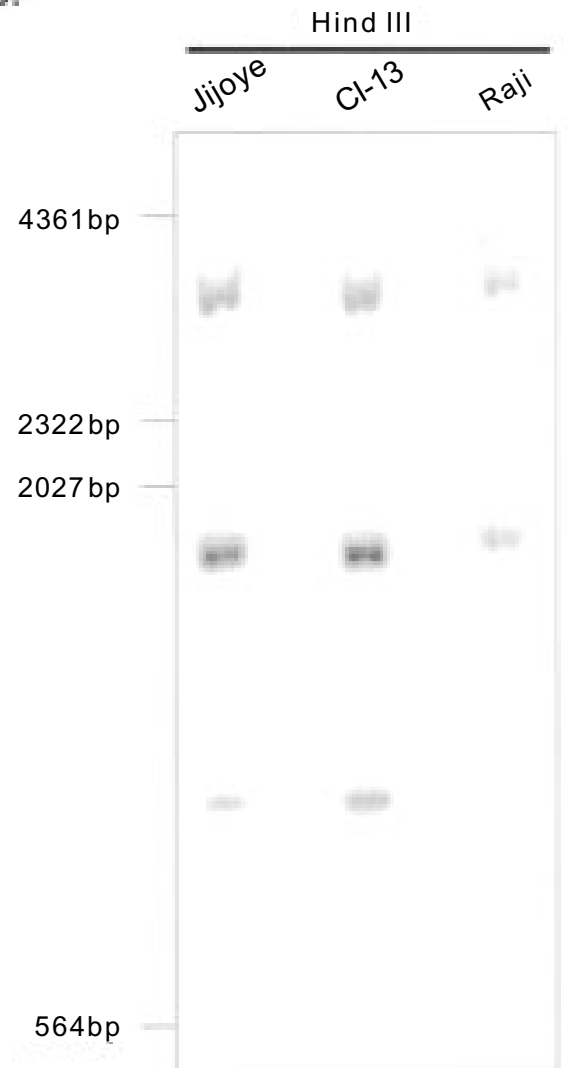

C.

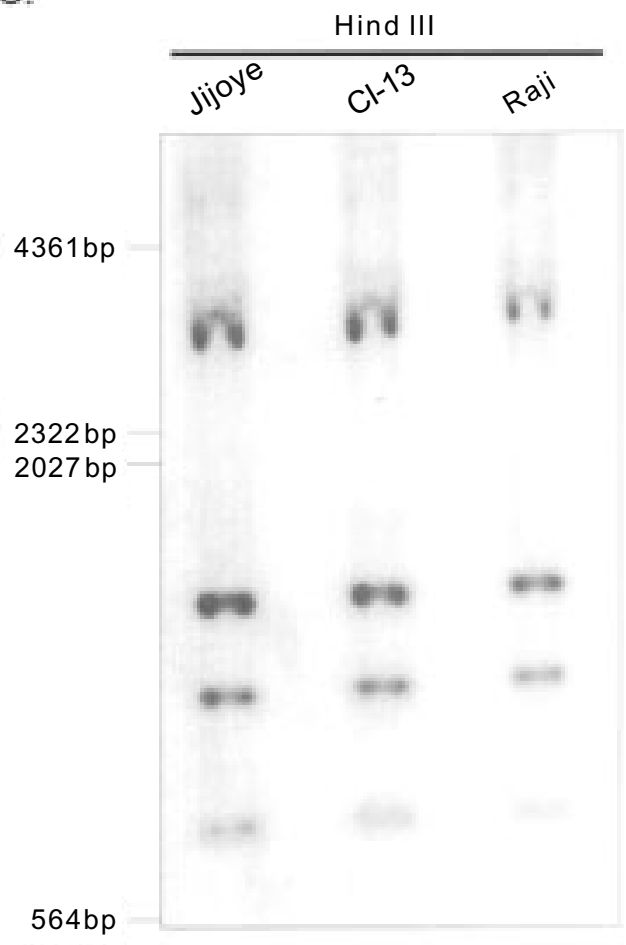

Fig. 5. Southern blot analysis of the $5^{\prime}$-flanking region of the BCL-6 gene in Jijoye and Clone-13. (A) Partial restriction maps of the BCL-6 gene 5'-flanking region. The positions of the probes for Southern blot analysis are indicated. Filled and empty boxes represent coding and noncoding exons, respectively. Restriction sites: B, BamH I; Bg, Bgl II; H, Hind III; R, EcoR I; S, Sac I; X, Xba I.(B) Southern blot analysis of BCL-6 gene in Jijoye and Clone-13 with Probe 1. High-molecular-weight DNA from Jijoye and Clone13 were digested with the indicated enzyme fractionated by agarose gel and transferred to membranes. (C) Southern blot analysis of BCL-6 gene with probe 2.

both cell lines. By sequence analysis, we did find multiple, biallelic mutations in this region of the BCL-6 gene in both cell lines. As summarized in Table 1, alterations include common and cellspecific single base pair substitutions, small deletions, and insertions.

We also scanned a large region of the BCL-6 gene promoter $-300 \mathrm{bp}$ to +3188 and did not detect any mutations. These sequencing results suggest that mutations cluster within the hot-spot region analyzed above, consistent with the existing literature.

Point Mutations in the First Intron do not Influence Reporter Gene Expression In Vitro

We subsequently determined the biallelic distribution of some of the mutations. First, fragments of 
A.

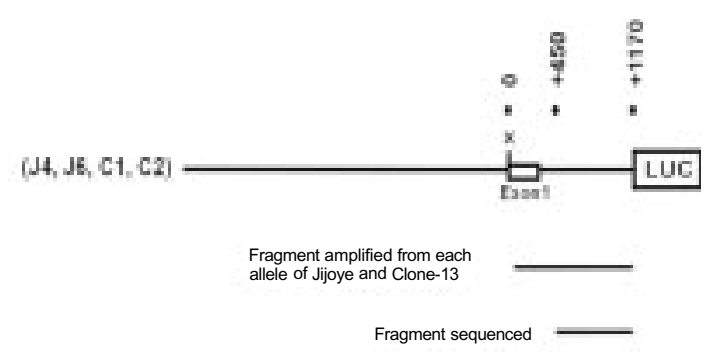

B.

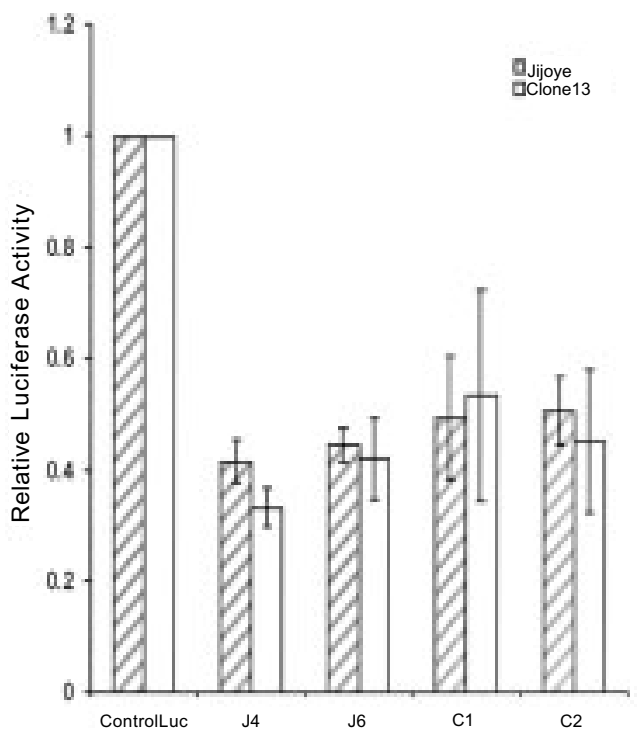

Fig. 6. Transfection data of plasmids with different alleles from Jijoye and Clone-13. Reporters were transfected into Jijoye and Clone-13 cells together with $\beta$-gal expression vector. Relative luciferase activity subtracted the background (pGL2-Basic) are represented as percentage of the pGL2-control vector value. The bars are shown as mean $\pm \operatorname{SD}(n>3)$.

B6S1/B6AS1 (+31 to $+1170 \mathrm{bp})$ were amplified from Jijoye and Clone-13 and then linked upstream of a $\sim 3-\mathrm{kb}$ BCL-6 promoter derived from plasmid pLA/B14.5 together with a downstream reporter gene (Fig. 6A). Multiple clones from each cell line were picked for sequence analysis using a sequencing primer starting from $+450 \mathrm{bp}$ and primer B6AS 1. The distribution of the mutations was separated into four types, corresponding to the four different alleles detected in the two cell lines (Table 2). $\mathrm{J} 4$ and $\mathrm{J} 6$ are derived from the two alleles from Jijoye; C1 and C2 are from Clone-13.

To determine if these mutations alter the transcription rate of the downstream gene, we transfected the above four constructs into Jijoye and Clone-13 cells and measured their transcription activities. As shown in Figure 6B, the relative luciferase activities of the four constructs overlapped in both cell lines, indicating that the mutations in the first intron do not have a significant influence on gene expression in vitro.

\section{Discussion}

We have previously identified a pair of isogenic human B lymphoblastoid cell lines (Jijoye and Clone-13) that have distinct profiles of class II major histocompatibility complex (MHC) gene expression (29). The Clone-13 cell line was derived from Jijoye, and expresses only HLA-DQ genes ( $\alpha$ and $\beta)$, whereas the Jijoye cell line expresses all three sets of class II genes (HLA-DR, DQ, and DP). Studies of these cell lines by cell fusion and transient transfection experiments have demonstrated that the lack of DR and DP gene expression in Clone-13 cells stems from a defect in a trans-acting factor influenced by the nature of a divergent class II cis-element termed the X2 box (36). Fusion with a panel of class II MHC mutant $B$ cell lines indicated the mutant gene in Clone-13 was identical to that found in another mutant cell line, RJ2.2.5 (37).

The affected gene in RJ2.2.5, Clone-13, and other members of the complementation group was identified by complementation cloning experiments (38). The polypeptide encoded by this factor, CIITA, functions as a master regulatory gene for antigen presentation, simultaneously activating several genes involved in immune recognition. Both alleles of the CIITA gene have been reported to harbor mutations in Clone-13 (30). However, the disparate

Table 1. Mutational analysis of the first intron of the BCL-6 gene in Jijoye and Clone-13 cell lines

\begin{tabular}{|c|c|c|}
\hline Cell Line & Mutations* & Polymorphisms \\
\hline Jijoye & $\begin{array}{l}\text { T/C(461); C/A(520); C/G(520); T/A(546); } \\
\text { G/C(644); G/C(703); T/C(848); T/G(914); C/G(1047) }\end{array}$ & +T (876) homozygous \\
\hline Clone-13 & $\begin{array}{l}\text { T/C(461); C/A(520); C/G(520); T/A(546); } \\
\text { T/C(669); G/C(703), } \Delta(\mathrm{CT}) / A(773)\end{array}$ & +T (876) homozygous \\
\hline
\end{tabular}

*position +1 refers to the first nucleotide of the BCL-6 cDNA (Migliazza et al, PNAS 92: 12520, 1995). All mutations found are in heterozygosis and confirmed in both strands. Cell type-specific mutations are bolded. 
Table 2. Biallelic distribution of the mutations in Jijoye and Clone-13

\begin{tabular}{llll}
\hline Cell Line & Allele & Construct Name & \multicolumn{1}{c}{ Mutations on Allele } \\
\hline Jijoye & allele 1 & $\mathrm{J} 4$ & $\mathrm{C} / \mathrm{A}(520), \mathrm{T} / \mathrm{A}(546), \mathrm{T} / \mathrm{A}(644), \mathrm{G} / \mathrm{C}(703), \mathrm{T} / \mathrm{G}(914), \mathrm{C} / \mathrm{G}(1047)$ \\
\multirow{3}{*}{ Clone-13 } & allele 2 & $\mathrm{J} 6$ & $\mathrm{~T} / \mathrm{C}(461), \mathrm{C} / \mathrm{G}(520), \mathrm{T} / \mathrm{C}(848)$ \\
& allele 1 & $\mathrm{C} 1$ & $\mathrm{~T} / \mathrm{C}(461), \mathrm{C} / \mathrm{G}(520), \mathrm{T} / \mathrm{C}(699), \Delta(\mathrm{CT}) / \mathrm{A}(733)$ \\
& allele 2 & $\mathrm{C} 2$ & $\mathrm{C} / \mathrm{A}(520), \mathrm{T} / \mathrm{A}(546), \mathrm{G} / \mathrm{C}(703)$
\end{tabular}

class II MHC phenotypes of RJ2.2.5 (pan-class II negative) and Clone-13 (-DQ positive) suggest that there are other isotype-restricted phenomena occurring in Clone-13 that are distinct from CIITA $(30,34)$.

One approach we used to identify factors involved in isotype-restricted MHC gene expression was to perform differential display on RNAs isolated from this and other isogenic cell pairs. This analysis would also be expected to potentially identify other genes regulated by CIITA (or other class II transactivators mutated in class II mutant B cells falling in other complementation groups).

One differentially expressed gene was found to be BCL- 6 cDNA. The product of the BCL-6 gene, a Kruppel-like zinc finger protein (1-3), has been the focus of intensive investigations in recent years. Structural alterations of the 5 ' noncoding region of the BCL-6 gene leading to the expression of a normal protein under the control of heterologous promoters have been found in $40 \%$ of DLCL and 5-10\% of follicular lymphomas (FL) $(1,15,39)$. The BCL-6 protein is predominantly expressed in germinal center $\mathrm{B}$ cells and related lymphomas, and also a small subpopulation of T cells (9-11). Most interestingly, transgenic experiments implicate BCL-6 play important roles in germinal center formation, and in the IL-4 signaling pathway $(22,23)$.

Our results clearly demonstrate that BCL-6 is differentially expressed in Jijoye and Clone-13. Previous studies have shown that BCL-6 can be regulated at transcriptional level as well as a posttranslational level through MAPK phosphorylation pathway $(40,41)$. The Northern and Western analyses, together with the results of nuclear run-on assay, strongly suggest that BCL- 6 expression is mainly controlled at the transcriptional level in Jijoye and Clone-13.

We did of course probe whether BCL-6 is regulated by CIITA or vice versa. This was done in two ways. First, we compared the expression profiles of CIITA, MHC class-II molecules, and BCL-6 in Jijoye/Clone-13 and another pair of B lymphoblastoid cell lines Raji/RJ2.2.5 (Table 3). Raji expresses CIITA, all class-II genes, and BCL-6, whereas its derivative RJ2.2.5 is CIITA-negative and class IInegative but BCL-6 positive (data not shown). From Table 3, no correlation is obvious between CIITA and BCL-6. Second, we directly asked whether ectopic expression of CIITA in CIITA-deficient HeLa cells could induce BCL-6 gene expression (and vice versa). Both experiments were negative (although control CIITA-dependent and BCL-6 repressed genes were induced or repressed as expected). Studies have shown that CIITA is regulated through the JAK 1-STAT1 pathway (42), and we have found that STAT1 is also involved in induction of BCL-6 transcription by IFN- $\gamma$ in Jurkat cells (unpublished data). Thus it is more likely that BCL-6 and CIITA are both early response genes during B-cell development, but do not crossregulate each other.

Studies have shown that promoter substitutions caused by chromosomal translocations may cause deregulation of BCL-6 in a number of DLCL cases (17). The majority of these breakpoints lie within the $5^{\prime}$ portion of the first intron, and in a few cases the breakpoints are located immediately $5^{\prime}$ to the promoter region (17). However, in our case, gross deletions of the BCL- 6 gene are not detected in these regions as shown by Southern blot analyses, and no promoter defects were found. Therefore, it is unlikely that the distinct expression of BCL-6 in the two cell lines is due to structural alterations of the gene.

Recent studies demonstrated that the BCL-6 gene accumulates large numbers of mutations in normal and malignant $B$ cells with a pattern similar to that seen in Ig genes (21). These mutations are independent of chromosomal rearrangements; and a stretch of $730 \mathrm{bp}$ region $\sim 100 \mathrm{bp}$ downstream the first noncoding exon, which also overlaps the major cluster of chromosomal breakpoints, was determined to have the most frequent mutations (20). It is

Table 3. Summary of expression status of CIITA, class-II molecules and BCL-6 in Jijoye/Clone13 and Raji/RJ2.2.5

\begin{tabular}{lcccc}
\hline & Jijoye & Clone13 & Raji & RJ2.2.5 \\
\hline CIITA & + & - & + & - \\
HLA-DP & + & - & + & - \\
HLA-DQ & + & + & + & - \\
HLA-DR & + & - & + & - \\
BCL-6 & - & + & + & + \\
& & & & \\
\hline
\end{tabular}


possible that this hot-spot region of mutations, together with the upstream BCL- 6 promoter, may be important in regulating BCL-6 transcription (20). In fact, we did find multiple, heterozygous mutations in this region, but these mutations do not affect gene expression as suggested by the results of the transient transfection experiments. The biological significance of the mutations is still unclear. We have not ruled out the possibility that (1) the mutations need to work in concert with downstream sequence to show their function on transcription or (2) there are mutations outside the region analyzed that can mediate transcription. Further experiments are required to elucidate the role of the somatic mutations in regulating BCL-6 expression.

In summary, we report a pair of B lymphoblastoid cell lines that exhibit distinct transcription of the BCL-6 gene. The multiple mutations in the first intron do not have effects on gene expression in vitro; promoter substitutions caused by chromosomal translocations are not detected in the two cell lines. Therefore, it is likely that the differences in transacting factor(s) explain the difference in BCL-6 transcription in these otherwise isogenic cell lines. Experiments comparing the occupancy of the promoter by trans-acting factors within the two cell lines are in progress to further map important regulatory mechanisms in BCL-6 gene expression. Preliminary data from these experiments suggest that the BCL-6 locus is occupied in both cell lines. This indicates that the differential expression of BCL-6 in the two cell lines is not due to differences in the general chromatin structure of the locus or to accessibility of the proximal promoter to binding by transcription factors. It is therefore likely that the difference in expression may be due to mutations in regulatory domains of these factors or to factors that are critical for transcription.

The existence of stable cell lines exhibiting distinct BCL-6 expression patterns should facilitate the elucidation of the regulatory mechanisms on the BCL-6 gene, just as they permitted the tremendous progress in research on class II MHC gene transcription over the past decade.

\section{Acknowledgments}

We are grateful to Professor Riccardo Dalla-Favera of Columbia University for advice and for some of the reagents used in these experiments. We also thank the members of the Ono laboratories at Harvard and University College London for helpful discussions and advice.

\section{References}

1. Ye BH, Rao PH, Chaganti RS, Dalla-Favera R. (1993) Cloning of bcl-6, the locus involved in chromosome translocations affecting band 3q27 in B-cell lymphoma. Cancer Res. 53: 27322735.

2. Ye BH, Lista F, Lo Coco F, et al. (1993) Alterations of a zinc finger-encoding gene, BCL-6, in diffuse large-cell lymphoma. Science 262: 747-750.
3. Chang CC, Ye BH, Chaganti RS, Dalla-Favera R. (1996) BCL-6, a POZ/zinc-finger protein, is a sequence-specific transcriptional repressor. Proc. Natl. Acad. Sci. U.S.A. 93: 6947-6952.

4. Dhordain P, Albagli O, Lin RJ, et al. (1997) Corepressor SMRT binds the BTB/POZ repressing domain of the LAZ3/BCL6 oncoprotein. Proc. Natl. Acad. Sci. U.S.A. 94: 10762-10767.

5. Wong CW, Privalsky ML. (1998) Transcriptional repression by the SMRT-mSin 3 corepressor: multiple interactions, multiple mechanisms, and a potential role for TFIIB. Mol. Cell Biol. 18: $5500-5510$.

6. Okabe S, Fukuda T, Ishibashi K, et al. (1998) BAZF, a novel Bcl6 homolog, functions as a transcriptional repressor. Mol. Cell Biol. 18: 4235-4244.

7. Davies JM, Hawe N, Kabarowski J, et al. (1999) Novel $\mathrm{BTB} / \mathrm{POZ}$ domain zinc-finger protein, $\mathrm{LRF}$, is a potential target of the LAZ-3/BCL-6 oncogene. Oncogene 18: 365-375.

8. Zhang W, Mi J, Li N, et al. (2001) Identification and characterization of DPZF, a novel human $\mathrm{BTB} / \mathrm{POZ}$ zinc finger protein sharing homology to BCL-6. Biochem. Biophys. Res. Commun. 282: 1067-1073.

9. Cattoretti G, Chang CC, Cechova K, et al. (1995) BCL-6 protein is expressed in germinal-center B cells. Blood 86: 45-53.

10. Fukuda T, Miki T, Yoshida T, et al. (1995) The murine BCL6 gene is induced in activated lymphocytes as an immediate early gene. Oncogene 11: 1657-1663.

11. Otsuki T, Yano T, Clark HM, et al. (1995) Analysis of LAZ3 (BCL-6) status in B-cell non-Hodgkin's lymphomas: results of rearrangement and gene expression studies and a mutational analysis of coding region sequences. Blood 85: 2877-2884.

12. Allman D, Jain A, Dent A, et al. (1996) BCL-6 expression during B-cell activation. Blood 87: 5257-5268.

13. Seyfert VL, Allman D, He Y, Staudt LM. (1996) Transcriptional repression by the proto-oncogene BCL-6. Oncogene 12: 2331-2342.

14. Reljic R, Wagner SD, Peakman LJ, Fearon DT. (2000) Suppression of signal transducer and activator of transcription 3dependent B lymphocyte terminal differentiation by BCL-6. J. Exp. Med. 192: 1841-1848.

15. Akasaka T, Ueda C, Kurata $M$, Akasaka $H$, Yamabe $H$, Uchiyama T, Ohno H. (2000) Nonimmunoglobulin (nonIg)/BCL6 gene fusion in diffuse large B-cell lymphoma results in worse prognosis than Ig/BCL6. Blood 96: 2907-2909.

16. Nakamura Y. (2000) Internal deletions within the BCL6 gene in B-cell non-Hodgkin's lymphoma. Leuk. Lymphoma 38: 505-512.

17. Qi CF, Hori M, Coleman AE, et al. (2000) Genomic organisation and expression of BCL6 in murine B-cell lymphomas. Leuk. Res. 24: 719-732.

18. Ariatti C, Vivenza D, Capello D, et al. (2000) Common-variable immunodeficiency-related lymphomas associate with mutations and rearrangements of BCL-6: pathogenetic and histogenetic implications. Hum. Pathol. 31: 871-873.

19. Muramatsu M, Akasaka T, Kadowaki N, et al. (1996) Rearrangement of the BCL6 gene in B-cell lymphoid neoplasms: comparison with lymphomas associated with BCL2 rearrangement. Br. J. Haematol. 93: 91 1-920.

20. Migliazza A, Martinotti S, Chen W, et al. (1995) Frequent somatic hypermutation of the $5^{\prime}$ noncoding region of the BCL6 gene in B-cell lymphoma. Proc. Natl. Acad. Sci. U.S.A. 92: 12520-12524.

21. Shen HM, Peters A, Baron B, Zhu X, Storb U. (1998) Mutation of BCL- 6 gene in normal B cells by the process of somatic hypermutation of Ig genes. Science 280: 1750-1752.

22. Dent AL, Shaffer AL, Yu X, Allman D, Staudt LM. (1997) Control of inflammation, cytokine expression, and germinal center formation by BCL-6. Science 276: 589-592.

23. Ye BH, Cattoretti G, Shen Q, et al. (1997) The BCL-6 protooncogene controls germinal-centre formation and Th2-type inflammation. Nat. Genet. 16: 161-170.

24. Pulvertaft RJ, Pulvertaft I. (1996) Spontaneous "transformation" of lymphocytes from the umbilical-cord vein. Lancet 2: 892-893.

25. Suzuki M, Hinuma Y. (1974) Evaluation of Epstein-Barr virus-associated nuclear antigen with various human cell lines. Int. J. Cancer 14: 753-761. 
26. Heston L, Rabson M, Brown N, Miller G. (1982) New EpsteinBarr virus variants from cellular subclones of P3J-HR-1 Burkitt lymphoma. Nature 295: 160-163.

27. Zhang X, Wu J, Gao D, et al. (1999) Cloning and sequencing of the fragment of angiostatin K (1-3) gene. Chin. Med. J. (Engl.) 112: 1013-1015.

28. Ono SJ, Colle E, Guttmann RD, Fuks A. (1989) Interferongamma induces transcription and differential expression of MHC genes in rat insulinoma cell line RINm5F. Diabetes 38: 911-916.

29. Ono SJ, Bazil V, Sugawara M, Strominger JL. (1991) An isotype-specific trans-acting factor is defective in a mutant $\mathrm{B}$ cell line that expresses HLA-DQ, but not -DR or -DP. J. Exp. Med. 173: 629-637.

30. Abdulkadir SA, Ono SJ. (1996) How are class II MHC genes turned on and off? FASEB J. 9: 1429-1435.

31. Tai AK, Zhou G, Chau K, Ono SJ. (1999) Cis-element dependence and occupancy of the human invariant chain promoter in CIITA-dependent and -independent transcription. Mol. Immunol. 36: 447-460.

32. Reith W, Mach B. (2001) The bare lymphocyte syndrome and the regulation of MHC expression. Annu. Rev. Immunol. 19: 331-373.

33. Mach B. (1999) Perspectives: immunology. Regulating the regulator. Science 285: 1367.

34. Guermonprez P, Valladeau J, Zitvogel L, Thery C, Amigorena S. (2002) Antigen presentation and T cell stimulation by dendritic cells. Annu. Rev. Immunol. 20: 62 1-667.

35. Rajeevan MS, Ranamukhaarachchi DG, Vernon SD, Unger ER. (2001) Use of real-time quantitative PCR to validate the results of cDNA array and differential display PCR technologies. Methods 25: 443-451.

36. Ono SJ, Song Z. (1995) Mapping of the interaction site of the defective transcription factor in the class II major histocompatibility complex mutant cell line clone-13 to the divergent X2-box. J. Biol. Chem. 270: 6396-6402.

37. Ono SJ, Liou HC, Davidon R, Strominger JL, Glimcher LH. (1991) Human X-box-binding protein 1 is required for the transcription of a subset of human class II major histocompatibility genes and forms a heterodimer with c-fos. Proc. Natl. Acad. Sci. U.S.A. 88: 4309-4312.

38. Yang Z, Accolla RS, Pious D, Zegers BJ, Strominger JL. (1988) Two distinct genetic loci regulating class II gene expression are defective in human mutant and patient cell lines. ЕМВО J. 7: 1965-1972.

39. Vitolo U, Botto B, Capello D, et al. (2002) Point mutations of the BCL-6 gene: clinical and prognostic correlation in B-diffuse large cell lymphoma. Leukemia 16: 268-275.

40. Kikuchi M, Miki T, Kumagai T, et al. (2000) Identification of negative regulatory regions within the first exon and intron of the BCL6 gene. Oncogene 19: 4941-4945.

41. Niu H, Ye BH, Dalla-Favera R. (1998) Antigen receptor signaling induces MAP kinase-mediated phosphorylation and degradation of the BCL-6 transcription factor. Genes Dev. 12: 1953-1961.

42. Reith W, Mach B. (2001) The bare lymphocyte syndrome and the regulation of MHC expression. Annu. Rev. Immunol. 19: 331-373. 